\title{
Terminal Sequence-Specific Interparticle Attraction between DNA Duplex-Carrying Polystyrene Microparticles in Aqueous Salt Solution Assessed by Optical Tweezers
}

\author{
Hiroya Nakauchi, ${ }^{1}$ Mizuo Maeda, ${ }^{1,2}$ and Naoki Kanayama ${ }^{1,2,3 *}$
}

1. Department of Biomedical Engineering, Graduate School of Medicine, Science and Technology, Shinshu University, 4-17-1 Wakasato, Nagano, Nagano 380-8553, Japan

2. Bioengineering Laboratory, RIKEN Cluster for Pioneering Research, 2-1 Hirosawa, Wako, Saitama 351-0198, Japan

3. Institute of Biomedical Science, Interdisciplinary Cluster for Cutting Edge Research, Shinshu University, 3-1-1 Asahi, Matsumoto, Nagano 390-8621, Japan

\section{Corresponding Author}

E-mail: nkanayama@shinshu-u.ac.jp,nkanayama@riken.jp (N.K.)

\section{Contents}

S-1. Base sequences of the fluorophore-labeled oligo-DNAs

$\mathrm{S}-2$. Interparticle attraction for the pair of s15-PS particles

S-3. Interparticle attraction for the pair of comp15/mis15(C)-PS particles

S-4. Interparticle attraction for the pair of comp15-PS particles in $\mathrm{MgCl}_{2}$ solution

S-5. Interparticle attraction for the pair of mis15(X)-PS particles at $40 \mathrm{mM} \mathrm{MgCl}_{2}$

S-6. Duplex formation of sample DNAs on the s15-PS surface in $\mathrm{MgCl}_{2}$ solution

S-7. Zeta potential for the dsDNA-PS particles in various salt solutions

S-8. Potential binding site of water molecules to the nucleobase pairs 
S-1. Base Sequences of the Fluorophore-Labeled Oligo-DNAs

Table S1. Base sequences of the fluorophore-labeled oligo-DNAs

\begin{tabular}{|c|c|}
\hline code & sequence $\left(5^{\prime}\right.$ to $\left.3^{\prime}\right)$ \\
\hline Cy3-s15-DNA-NH ${ }_{2}$ & 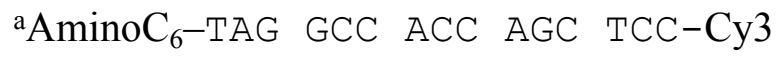 \\
\hline \multicolumn{2}{|l|}{ 5'-FAM sample DNA } \\
\hline 5'-FAM-comp15 & FAM-GGA GCT GGT GGC CTA \\
\hline 5'-FAM-mis15(C) & FAM-ĆGA GCT GGT GGC CTA \\
\hline 5'-FAM-mis15(A) & FAM- $\underline{A} G A$ GCT GGT GGC CTA \\
\hline 5'-FAM-mis15(T) & FAM-TGA GCT GGT GGC CTA \\
\hline
\end{tabular}

a. AminoC 6 : aminohexyl terminal $\left(\mathrm{H}_{2} \mathrm{~N}-\left(\mathrm{CH}_{2}\right)_{6}-\right)$. The underlined bases make unpaired (mismatched) terminals after the duplex formation with s15-DNA. 


\section{S-2. Interparticle Attraction for the Pair of s15-PS Particles}

(a)

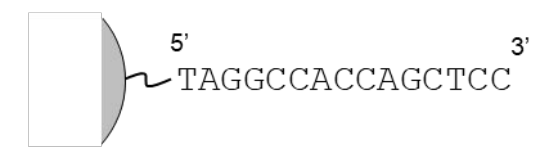

(b)

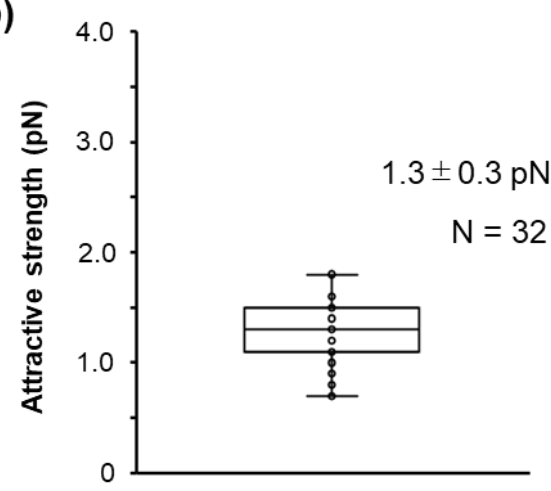

Figure S1. (a) Base sequence of an oligo-DNA strand (s15-DNA) immobilized on the s15-PS particle. (b) Boxplot of the maximum interparticle attraction observed on the $F-D$ curves for the pair of s15-PS particles on approach in $10 \mathrm{mM}$ MES buffer ( $\mathrm{pH}$ 7.2) containing $0.01 \mathrm{wt} \%$ Tween 20 and $750 \mathrm{mM} \mathrm{NaCl}$. The line in the box represents the median value. The force value is presented as the mean $\pm \mathrm{SD}$. 


\section{S-3. Interparticle Attraction for the Pair of comp15/mis15(C)-PS Particles}

(a)

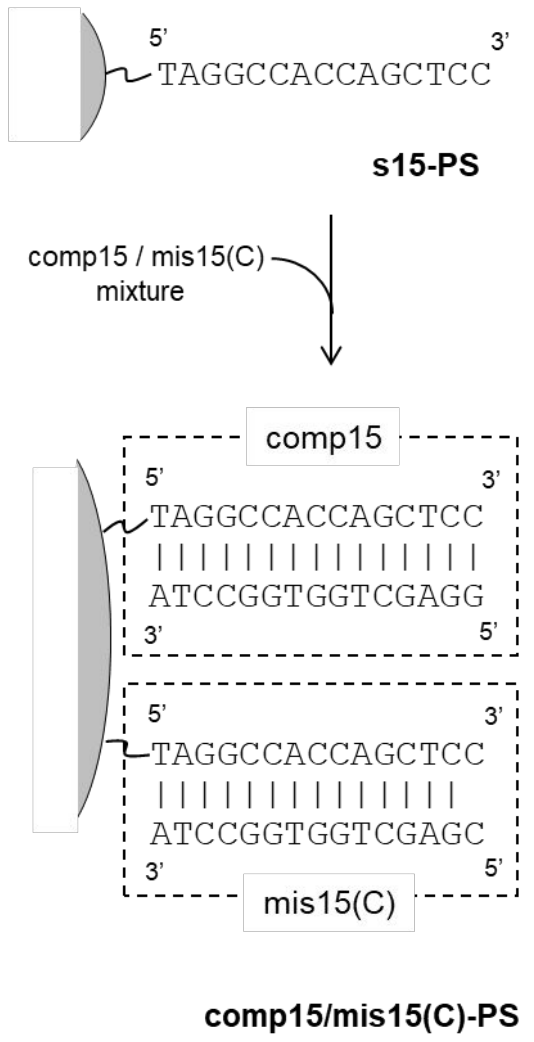

(b)
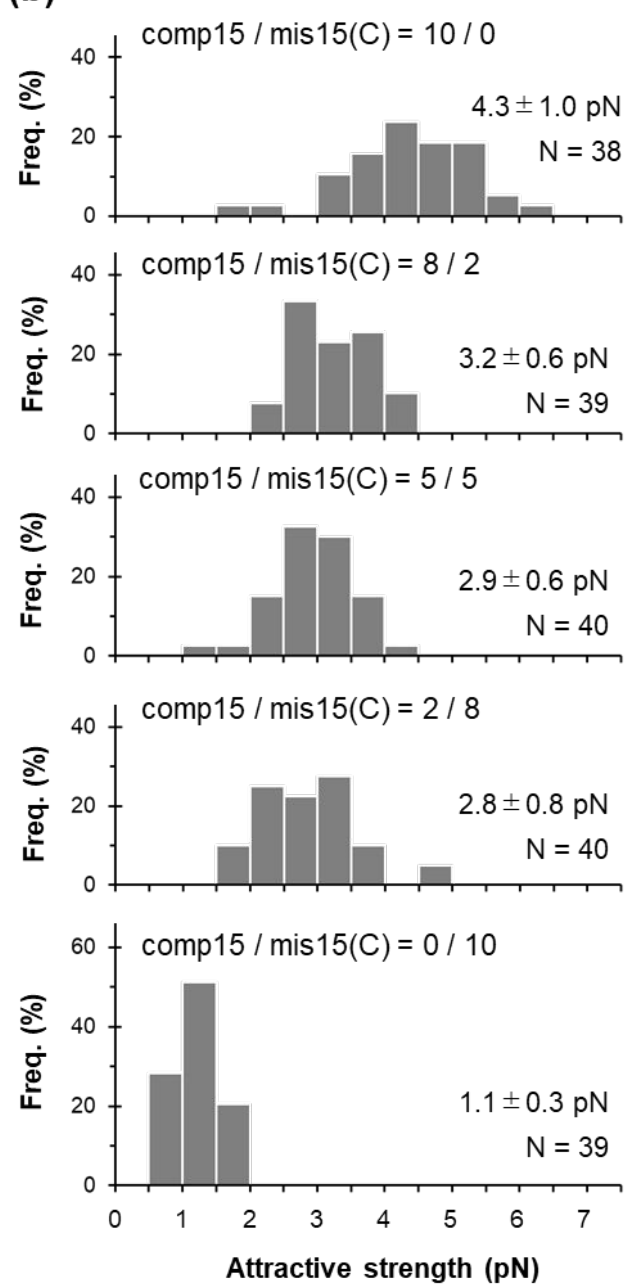

Figure S2. (a) Base sequences of duplexes formed on the comp $15 / \mathrm{mis} 15(\mathrm{C})$-PS. (b) Histograms for the maximum interparticle attraction distribution observed on the $F-D$ curves for the pairs of comp15/mis15(C)-PS particles on approach in $10 \mathrm{mM}$ MES buffer (pH 7.2) containing $750 \mathrm{mM} \mathrm{NaCl}$ and $0.01 \mathrm{wt} \%$ Tween 20 . To prepare a series of samples, the ratio of comp15/mis15(C) mixture was varied as $10 / 0,8 / 2,5 / 5,2 / 8$, and $0 / 10$. The total concentration of sample DNA (comp15 and mis15(C)) was fixed at $4.0 \mu \mathrm{M}$. All of the force values are presented as the mean $\pm \mathrm{SD}$. 
S-4. Interparticle Attraction for the Pair of comp15-PS Particles in $\mathrm{MgCl}_{2}$ Solution

(a)

(b)
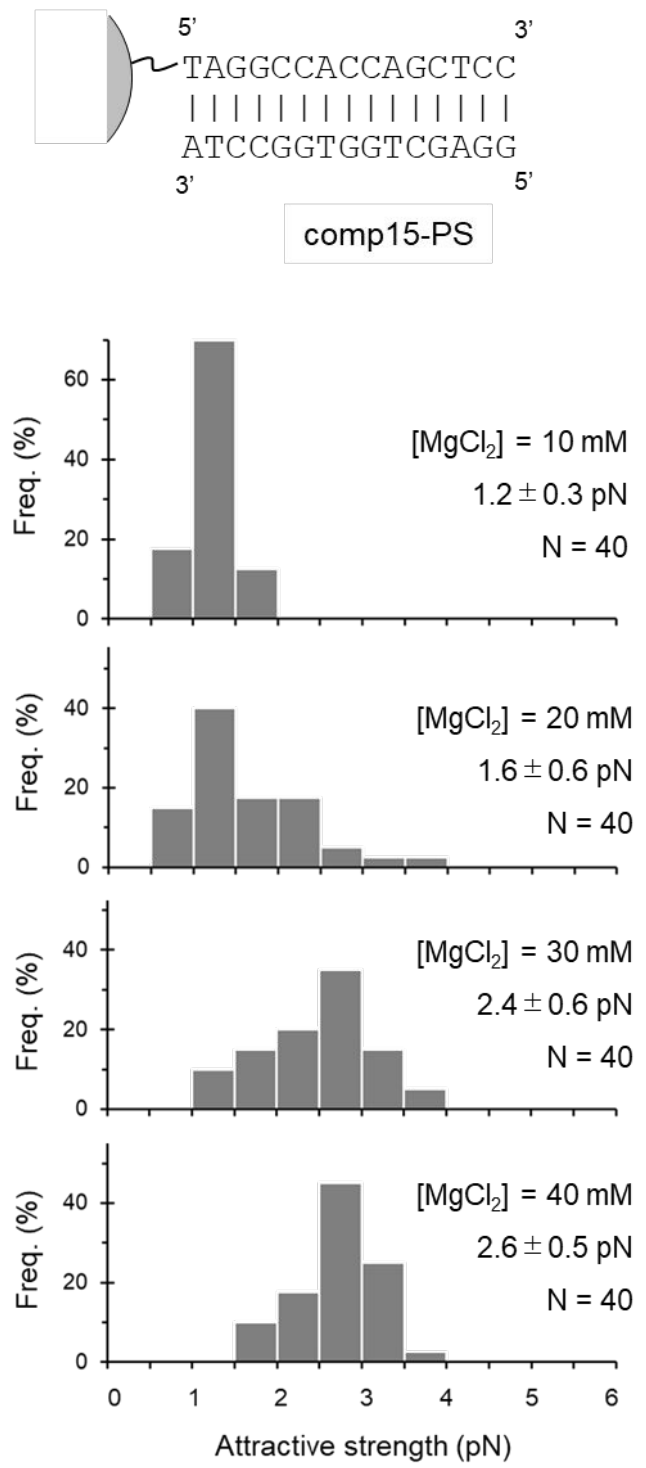

Figure S3. (a) Base sequence of the duplex formed on the comp15-PS. (b) Histogram of the maximum interparticle attraction observed on the $F-D$ curves for the pair of comp15PS particles on approach in $10 \mathrm{mM}$ MES buffer ( $\mathrm{pH} 7.2$ ) containing $0.01 \mathrm{wt} \%$ Tween 20 and various concentrations of $\mathrm{MgCl}_{2}$. All of the force values are presented as the mean \pm SD. 
S-5. Interparticle Attraction for the Pair of mis15(X)-PS Particles at $40 \mathbf{m M ~ M g C l}_{2}$

(a)

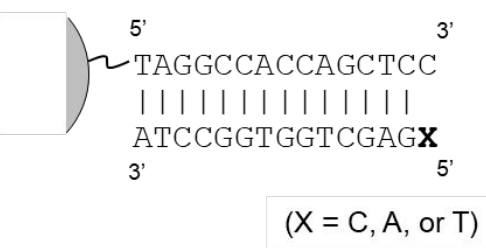

(c)

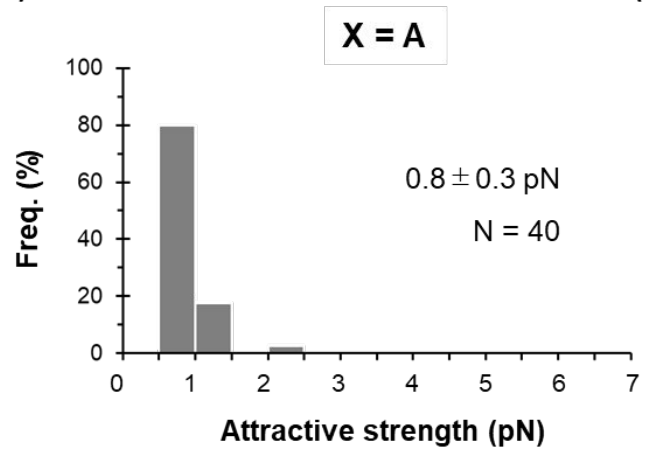

(b)

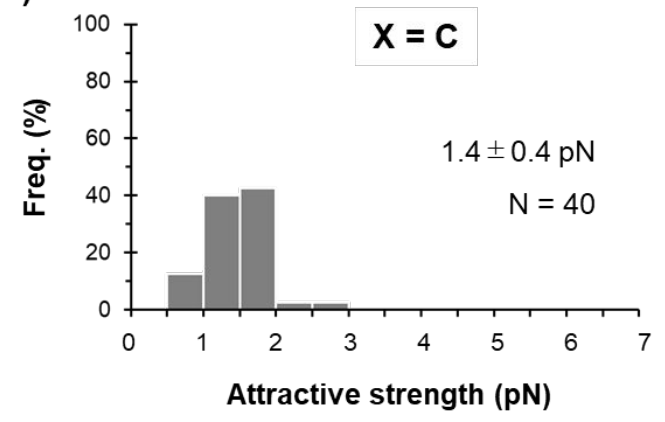

(d)

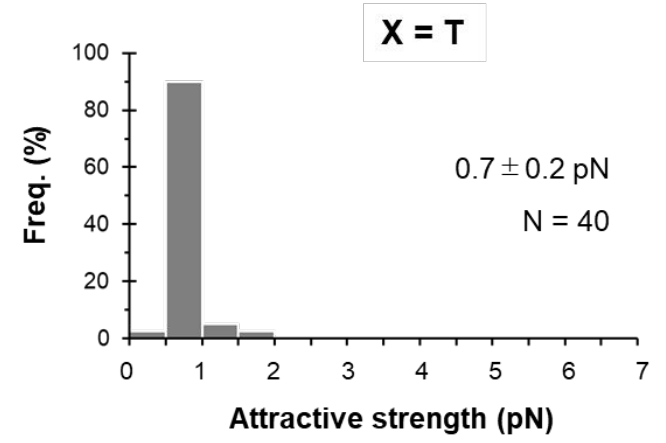

Figure S4. (a) Base sequence of the duplex formed on the mis15(X)-PS. (b-d) Histograms of the maximum interparticle attraction observed on the $F-D$ curves for the pairs of mis15(X)-PS particles on approach in $10 \mathrm{mM}$ MES buffer (pH 7.2) containing $0.01 \mathrm{wt} \%$ Tween 20 and $40 \mathrm{mM} \mathrm{MgCl}_{2}$ : (b) $\mathrm{X}=\mathrm{C}$, (c) $\mathrm{X}=\mathrm{A}$, and (d) $\mathrm{X}=\mathrm{T}$. All of the force values are presented as the mean $\pm \mathrm{SD}$. 
S-6. Duplex Formation of Sample DNAs on the s15-PS Surface in $\mathrm{MgCl}_{2}$ Solution

Table S2. Duplex formation of comp15 strands on the s15-PS surface in $\mathbf{M g C l}_{2}$ solution

\begin{tabular}{ccc}
\hline $\begin{array}{c}{\left[\mathrm{MgCl}_{2}\right]} \\
(\mathrm{mM})\end{array}$ & $\begin{array}{c}{ }^{\mathrm{a}} N_{\text {duplex }} \times 10^{-4} \\
(/ \text { particle })^{\mathrm{c}}\end{array}$ & $\begin{array}{c}{ }^{\mathrm{b}} P \\
(\%)^{\mathrm{c}}\end{array}$ \\
\hline 10 & $5.2 \pm 0.2$ & $4.3 \pm 0.2$ \\
20 & $3.9 \pm 1.6$ & $3.3 \pm 1.3$ \\
30 & $6.7 \pm 0.8$ & $5.6 \pm 0.7$ \\
40 & $3.7 \pm 1.2$ & $3.1 \pm 1.0$ \\
\hline
\end{tabular}

a. Number of duplexes formed on the resulting comp15-PS surface.

b. Percentage of the s15-DNA strands forming a duplex with comp15 on the s15-PS surface.

c. mean $\pm \mathrm{SD}(\mathrm{n}=3)$.

Table S3. Duplex formation of mis15(X) strands on the s15-PS surface at $40 \mathrm{mM}$ $\mathrm{MgCl}_{2}$

\begin{tabular}{ccc}
\hline & $\begin{array}{c}{ }^{\mathrm{a}} N_{\text {duplex }} \times 10^{-4} \\
(/ \text { particle })^{\mathrm{c}}\end{array}$ & $\begin{array}{c}{ }^{\mathrm{b}} P \\
(\%)^{\mathrm{c}}\end{array}$ \\
\hline $\operatorname{mis} 15(\mathrm{C})$ & $5.9 \pm 2.1$ & $4.9 \pm 1.8$ \\
$\operatorname{mis} 15(\mathrm{~A})$ & $4.9 \pm 0.8$ & $4.1 \pm 0.7$ \\
$\operatorname{mis} 15(\mathrm{~T})$ & $6.2 \pm 1.4$ & $5.2 \pm 1.2$ \\
\hline
\end{tabular}

a. Number of duplexes formed on the resulting mis15(X)-PS surface.

b. Percentage of the s15-DNA strands forming a duplex with mis15(X) on the s15-PS surface.

c. mean $\pm \mathrm{SD}(\mathrm{n}=3)$.

Hybridization conditions: [s15-PS] $=1.4 \times 10^{8}$ particles $/ \mathrm{mL}$, [sample DNA] $=4.0 \mu \mathrm{M}$ in $10 \mathrm{mM}$ MES buffer ( $\mathrm{pH}$ 7.2) containing $0.01 \mathrm{wt} \%$ Tween 20 and predetermined concentrations of $\mathrm{MgCl}_{2}(10,20,30$, and $40 \mathrm{mM})$, overnight in the dark at $25^{\circ} \mathrm{C}$. 


\section{S-7. Zeta Potential for the dsDNA-PS Particles in Various Salt Solutions}

The zeta potentials of the dsDNA-PS particles $\left(1.1 \times 10^{6}\right.$ particles $\left./ \mathrm{mL}\right)$ dispersed in the aqueous monovalent $\mathrm{NaCl}$ or divalent $\mathrm{MgCl}_{2}$ containing MES buffer (10 mM, pH 7.2) were determined by the electrophoretic light scattering using an ELS-Z zeta-potential and particle size analyzer (Otsuka Electronics Co., Osaka, Japan). The sample temperature was set at $25^{\circ} \mathrm{C}$. The measured electrophoretic mobility (v) was converted into the zeta potential $(\zeta)$ by applying the Smoluchowski equation: $\zeta=4 \pi \eta v / \varepsilon$, in which $\eta$ is the viscosity and $\varepsilon$ is the dielectric constant of the solvent, respectively.

(a)

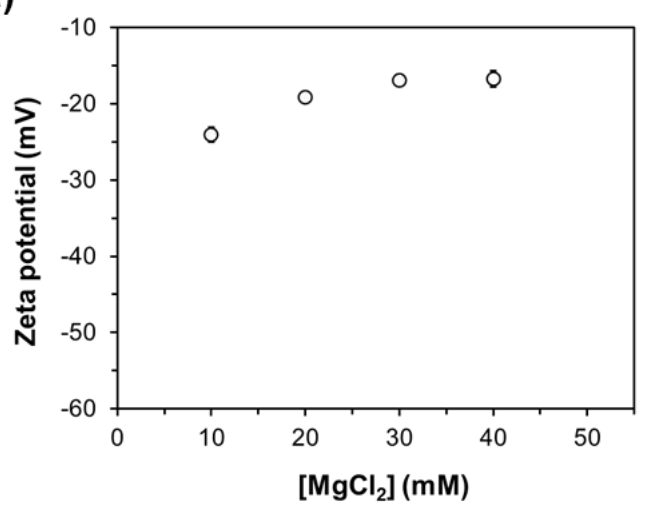

(b)

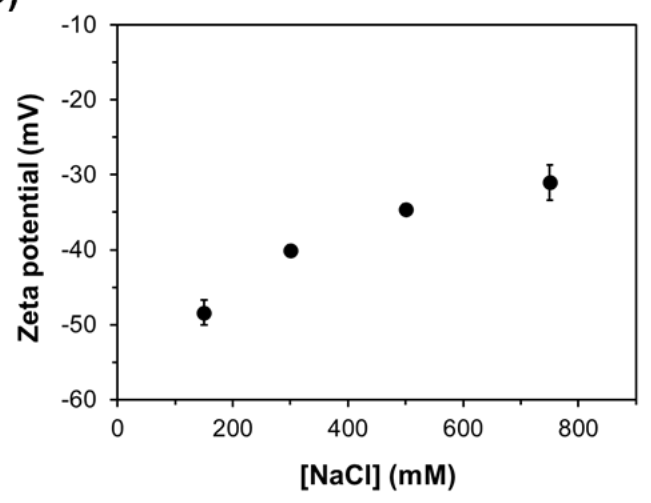

Figure S5. Zeta potential of the comp15-PS particle measured in $10 \mathrm{mM}$ MES buffer (pH 7.2) containing (a) $10-40 \mathrm{mM}$ of $\mathrm{MgCl}_{2}$ or (b) $150-750 \mathrm{mM}$ of $\mathrm{NaCl}$. Zeta potentials are presented as the mean $\pm \operatorname{SD}(n=5)$. 

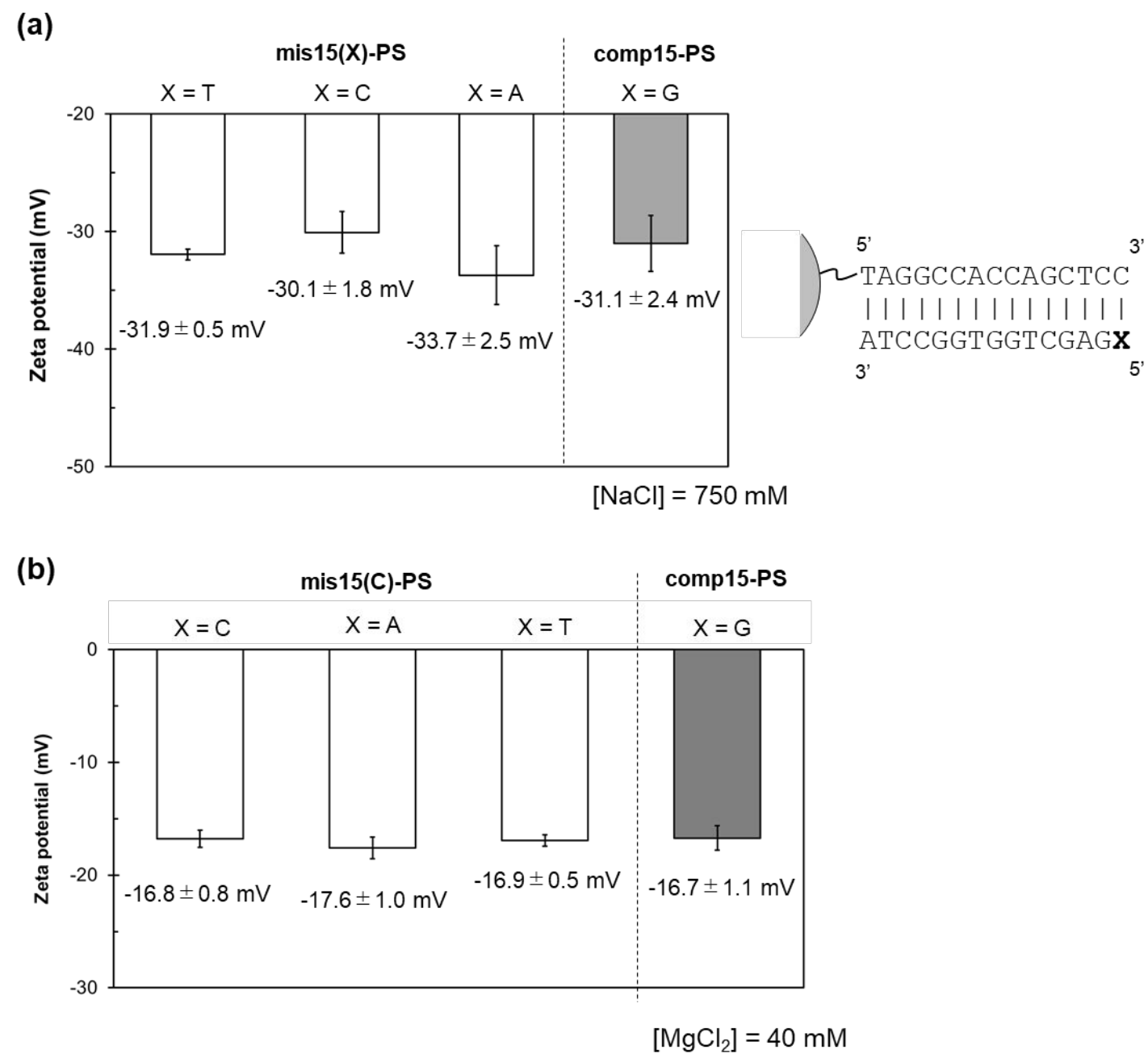

Figure S6. Zeta potential of the dsDNA-PS particles with different duplex terminals in $10 \mathrm{mM}$ MES buffer (pH 7.2) containing (a) $750 \mathrm{mM}$ of monovalent $\mathrm{NaCl}$ or (b) $40 \mathrm{mM}$ of divalent $\mathrm{MgCl}_{2}$. All of the zeta potential values are presented as the mean $\pm \mathrm{SD}(\mathrm{n}=$ 5). Note that the interparticle attraction was confirmed only for the $X=G$ (comp15-PS) under both salt conditions. 


\section{S-8. Potential binding site of water molecules to the nucleobase pairs}

(a) C-G pairing

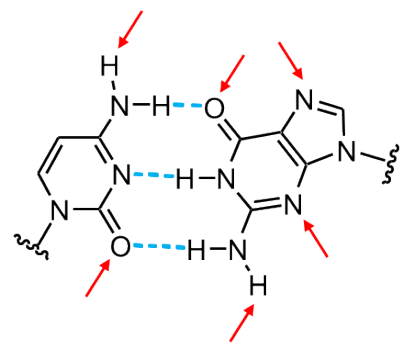

Number of binding site: 6

(c) C-C unpairing

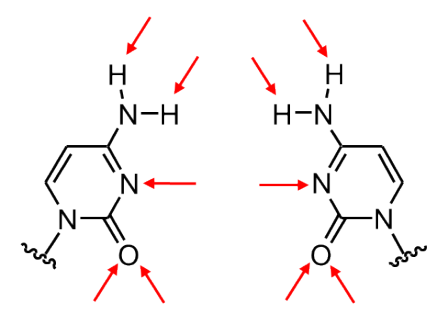

Number of binding site: 10 (b) C-A unpairing

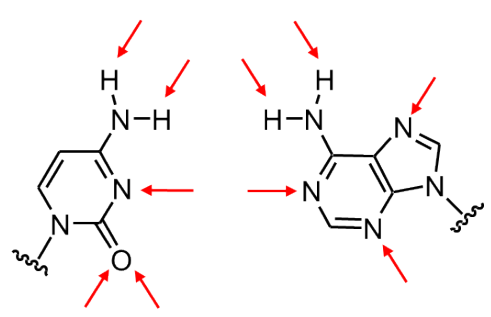

Number of binding site: 10

(d) C-T unpairing

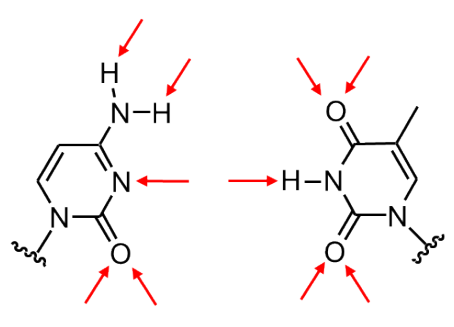

Number of binding site: 10

Figure S7. Potential binding sites of water molecules to the (a) $\mathrm{C}-\mathrm{G}$ pairing, (b) $\mathrm{C}-\mathrm{A}$ unpairing, (c) $\mathrm{C}-\mathrm{C}$ unpairing, and (d) $\mathrm{C}-\mathrm{T}$ unpairing nucleobase pairs. Red arrow demotes the potential binding site of water molecules. 